\title{
Modeling human pregnancy-associated developmental neurotoxicity in vitro using pluripotent stem cell-derived neurons and astrocytes
}

\author{
Xian Wu $\mathbf{W}^{1,2}$, Raymond Swetenburg ${ }^{2}$, Forrest Goodfellow ${ }^{1,2}$, Steven L. Stice ${ }^{1,2 *}$ \\ 'Interdisciplinary Toxicology Program, University of Georgia, Athens, GA 30602, United States \\ ${ }^{2}$ Regenerative Bioscience Center, University of Georgia, Athens, GA 30602, United States
}

Article Info

\section{Article Notes}

Received: December 13, 2017

Accepted: February 09, 2018

\section{${ }^{*}$ Correspondence:}

Dr. Steven L. Stice, ADS Complex, University of Georgia Athens, GA 30602; E-Mail: sstice@uga.edu;

(c) 2018 Stice S.L. This article is distributed under the terms of the Creative Commons Attribution 4.0 International License.

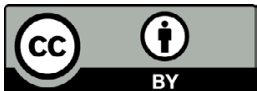

\section{Keywords}

Developmental neurotoxicity

Pluripotent stem cell

High throughput screening

Neural progenitor cell

Neurite outgrowth

Astrocyte

\section{ABSTRACT}

Developmental neurotoxicity is any toxic effect on the developing nervous system interfering with normal nervous system structure or function before or after birth and can be associated with neurodevelopmental disorders such as autism, attention deficit disorder, mental retardation and cerebral palsy. Currently, developmental neurotoxicity testing relies heavily on neurobehavioral and neuropathological studies in rodent models; however, an existing backlog of chemicals lack appropriate neurotoxicity information due to the low throughput nature and subsequent high cost of in vivo animal testing. Alternative models have been developed to replace rodent models (including non-mammalian models, brain slice culture, and primary and transformed cell models) though limitations to increasing throughput and reproducibility remain. In this review, we focus on the use of human pluripotent stem cells as a cell source for in vitro chemical screening. Pluripotent stem cell-based assays are derived from a virtually unlimited and uniform cell source making studies highly reproducible and relatively low cost compared to rodent models. Moreover, directed differentiation to distinct neural lineages, including neurons and astrocytes, provides isogenic, precise and tunable cell mixtures to mimic in vivo brain composition for high throughput screening. Thus, human pluripotent stem cell-derived neural models are poised to vastly increase throughput and decrease costs for developmental neurotoxicity screening.

\section{Introduction}

The pre- and post-natal brain is highly vulnerable to external insults from environmental factors, including some which have been linked to the development and progression of various central nervous system (CNS) disorders ${ }^{1}$. The resulting mental deficits, such as autism, learning disabilities and developmental delays, are likely due to a continuum of exposure during specific windows of susceptibility (WOS) in early human development ${ }^{1}$. Currently, little data exists relative to the vast number of chemicals and agents humans are exposed to either chronically (e.g. additives in manufacturing or agriculture), in specific geographic regions (e.g. air or water supply contamination) or during a given temporal period (e.g. chemical spills, smoking while pregnant). Developmental neurotoxicity (DNT) is a blanket term for any acute or chronic exposure to an agent during the course of CNS development causing an adverse outcome either immediately or later in life ${ }^{2}$.

Proper CNS development requires the precise spatial and temporal coordination of cell division, migration, differentiation and 
maturation, spanning almost the entire rostrocaudal axis of the human embryo ${ }^{3}$. Human CNS development begins with the specification of the neuroectodermal plate, which folds to form the neural tube at approximately three weeks gestation $^{3}$. Failure of the neural tube to close results in anencephaly or spina bifida ${ }^{4}$. Upon closure of the neural tube, cells are patterned based on their spatial position and divide and differentiate into the highly interconnected and interdependent regions of the human CNS. The CNS is still undergoing programmed changes even as late as adolescence, when synaptic pruning emphasizes certain neuronal pathways while deemphasizing others ${ }^{5}$ and faulty synaptic pruning has been strongly linked to autism ${ }^{6}$. Thus, the overall WOS for DNT spans well over a decade: from the beginnings of in utero development (e.g. spina bifida) up until pubescence (e.g. autism spectrum disorder), including the possibility that some insults may persist "silently" into adulthood, manifesting as Parkinson's or Alzheimer's disease ${ }^{7}$. It is therefore critical that appropriate models are developed which can mimic specific WOS and the human condition in a high-throughput, rapid and cost-effective manner.

Human pluripotent stem cells (PSC) can be expanded without limit in culture while remaining pluripotent, and offer a potential new tool for improved understanding of chemically-induced adverse reactions on neural development. Human PSC can theoretically become any cell in the body through biologically relevant signaling and follow a similar chronology. Therefore, the differentiation process is capable of modeling almost any stage of development, including WOS identified as critical to DNT. The advent of induced pluripotent stem cell (iPSC) technology, patient-specific iPSC can be differentiated to neural stem cells (NSC), neurons, and astrocytes. These derived cells not only offer a source of isogenic cell types ${ }^{8}$, but also enable the inclusion of cellular diversity for neurotoxicity screens ${ }^{9}$.

Neural stem cells can be isolated and expanded as an enriched population for DNT study

Human NSC isolated from neural rosettes can be maintained in culture with little to no phenotypic variation for extended periods of time thus providing an unlimited source of cells for DNT assays ${ }^{10}$. Self-renewal of human NSC are facilitated by multiple signaling pathways, including leukemia inhibiting factor (LIF) and fibroblast growth factor 2 (FGF2) ${ }^{11}$. These cells demonstrate an increased proliferation rate when LIF and FGF2, both individually and in combination, are added to growth medium ${ }^{12}$. The role of FGF2 is critical to NSC maintenance as it plays two distinct roles during early embryonic neural specification: early, it induces a "pro" neural state, then later it both promotes NSC proliferation (maintenance of the cell cycle) while antagonizing bone morphogenetic protein (BMP) to stabilize neural identity ${ }^{13,11}$. LIF enhances in vitro survival of human embryonic stem cell-derived NSC by reducing caspase-mediated apoptosis and both spontaneous and $\mathrm{H}_{2} \mathrm{O}_{2}$-induced reactive oxygen species ${ }^{14}$. LIF has been shown to bind the heterodimeric receptor of leukemia inhibiting factor receptor (LIFR) and glycoprotein 130 (gp130) ${ }^{14}$. Activation of gp130 is accompanied by the recruitment of activated signal transducer and activator of transcription 3 (STAT3) and subsequent activation of the phosphatidylinositol 3-kinase/protein kinase B (PI3K/Akt) and extracellular signal-regulated kinases (ERK) pathways, all of which are involved in the promotion of neuronal cell division and migration ${ }^{15}$.

The balance between survival and apoptosis during development helps regulate the size and shape of the nervous system. Insults to the expansion periods of NSC in vivo can cause devastating downstream effects later in development ${ }^{16}$. Human NSC have been utilized as an in vitro model to mimic critical aspects of progenitor cell function and to interrogate the disruption of these processes. For example, NSC are reproducibly susceptible to a set of known neurotoxic chemicals as is evident by caspase- $3 / 7$ activity and are suitable for future early-stage DNT testing ${ }^{17}$.

Thus, NSC could serve as an in vitro platform for DNT endpoints linked to the key processes of brain development including cell proliferation, migration, neurite outgrowth and neuronal/glial differentiation in neurotoxicity screens. Chemicals that interact with the cell cycle, cytoskeleton or calcium signaling would preferentially induce cellular changes, such as in proliferation and neurite outgrowth, associated with different WOS.

\section{Neurite outgrowth assays used in DNT studies}

Neurite outgrowth is a fundamental process of neuronal development, requiring membrane expansions and cytoskeletal dynamics ${ }^{18}$. The growth of axonal and dendritic processes (collectively called neurites) during brain development is a critical determinant of neuronal connectivity and disruption of this process can lead to cognitive deficits ${ }^{19}$. Recent advances in automated highcontent neurite outgrowth screening have been adapted for several cell lines, demonstrating neurite outgrowth assay efficacy for large scale screening and reliable data output.

Individual neurons extend a set of neurites that follow chemical guideposts toward appropriate target regions. Specifically, neurite outgrowth is controlled by complex interactions between adhesion molecules and their receptors, the cytoskeleton, intracellular calcium concentrations, and second messenger systems including protein kinases and phosphatases, making this process susceptible to a wide variety of insults ${ }^{20,21}$. Nerve outgrowth is led by the growth cone, the most distal 
structure to the cell body, which moves by the elongation and contraction of microfilament-containing filopodia known as microspikes ${ }^{22}$. Disruption of actin in microspikes inhibits further advancement of the dendrites and axon ${ }^{23}$. Axons in the developing neuron may extend for several feet prior to synapse formation ${ }^{23}$. The axonal structure is both supported and mediated by microtubules while the apical shape is changed by microfilaments ${ }^{24}$.

Harrill and coworkers (2010) characterized the morphological phenotype of human NSC-derived neurons cryopreserved at 14 days post-FGF2 withdrawal for use in an automated high-content image analysis assay to measure neurite outgrowth in vitro ${ }^{25}$. At 24 hours post-plating, these neurons expressed protein markers indicative of a neuronal phenotype, including Nestin, TUJ1, microtubule-associated protein 2 (MAP2), and phosphorylated neurofilaments. Concentration-dependent decreases in neurite outgrowth and ATP-content were observed following treatment with bisindolylmaleimide I (Bis-1) as well as other inhibitors ${ }^{25}$. In another study, neurite outgrowth measurements indicated the same cell types were more sensitive than rat cortical cultures to chemicals previously shown to inhibit neurite outgrowth ${ }^{26}$. Thus, neurite outgrowth in differentiating NSC represents a sensitive endpoint for non-cytotoxic agent exposure and is capable of predicting low dose neurotoxicity and interspecies differences ${ }^{26,27}$.

A recent study in our lab indicates neurite outgrowth of cryopreserved NSC-derived neurons can detect neurotoxic effects of chronic low-level exposure with higher sensitivity than high-level acute exposure for Bis-1, testosterone, and $\beta$-estradiol ${ }^{28}$. After two weeks exposure, we demonstrated that neurite outgrowth is inhibited by Bis-I at non-cytotoxic exposure levels after 14 days. This is one example in which neurite outgrowth and maturation were combined to assess chronic exposure neurotoxicity during an early WOS, spanning maturation of human NSC toward neurons ${ }^{28}$. Taken together, neurite outgrowth assays on NSC-derived cells are a highly sensitive endpoint capable of recapitulating both acute and chronic exposure during the biologically relevant periods of initial neurogenesis and neuronal maturation.

Importance of astrocytes and their derivation from NSC by epigenetic modulation

The human brain consists of more than $10^{11}$ neurons associated with over $10^{12}$ glial cells ${ }^{29}$. These glial cells maintain homeostasis in the brain by providing neurons with energy and substrates for neurotransmission ${ }^{30}$. Among the glial cell population, astrocytes are now considered far more active than were previously thought and are powerful instructors of synapse formation, function, plasticity and elimination, both in health and disease $\mathrm{e}^{31}$. Astrocytes secrete many neuroprotective factors including nerve growth factor (NGF), glial cell line-derived neurotrophic factor (GDNF) and brain-derived neurotrophic factor (BDNF) ${ }^{32}$. Additionally, astrocyte-sourced non-diffusible proteins, such as N-cadherin, NCAM, and integrins, can stabilize neurons.

Another important function of astrocytes is active detoxification of xenobiotics. In the brain, astrocytes express various cytochrome P450 (CYP) enzymes ${ }^{33}$, much like the liver. These enzymes are reported to be active at relatively high levels in astroglial cells and may play a critical role in the biotransformation of endogenous or exogenous compounds after they cross the blood-brain barrier $^{34}$. However, biotransformation of xenobiotics can also yield toxic reactive metabolites. For example, primary cultures of mouse brain astrocytes showed that one nearly universally expressed CYP isoform altered the toxic bioactivation of the anticonvulsant drug phenytoin, indicating the critical role of astrocytes in future DNT and drug development studies ${ }^{33}$.

Normally, human NSC are resistant to astrocytogenesis and show a strong neurogenic bias, similar to NSC from the early embryonic $\mathrm{CNS}^{11,35}$. Notably, several astrocytic loci are hypermethylated in human NSC, including a critical CpG site within the STAT3 recognition sequence in the promoter for the astrocytic marker glial fibrillary acidic protein (GFAP) ${ }^{35}$. However, after epigenetic modulation, NSC express prominent astrocytic markers in as little as five days of differentiation under BMP and LIF conditions ${ }^{35}$. In conjunction with demethylating agents, LIF signaling activates the downstream janus kinase (JAK)-signal transducer and activator of transcription (STAT) pathway, which is then able to bind the newly demethylated CpG site in the GFAP promoter ${ }^{36}$. BMP contributes to the expression of astrocyte-specific genes via the downstream formation of a SMAD1/STAT complex ${ }^{36}$. Therefore, it is possible to selectively generate either neurons or astrocytes from the same progenitor source in a rapid manner. Additionally, the derivation and subsequent maintenance of astrocytes utilize the same basal media as neuronal cultures thus making both cell types amenable to co-culture ${ }^{35,37}$.

A murine astrocyte-neuron co-culture model has previously demonstrated the impact of secreted astrocytic factors on neurons including neuronal survival, synapse formation, and plasticity ${ }^{38}$. As noted, NSC-derived astrocytes and neurons would facilitate a limitless, lineage-restricted cell source for the generation of neuronal subtypes and supporting glial cells for high-throughput DNT screening ${ }^{39}$. Additionally, stem cell-derived models reduce uncertainty in toxicity testing caused by extrapolation of data derived from animal tissue to humans ${ }^{25}$. Unlike primary cultures that contain multiple cell types that may vary widely among cell preparation made from tissues, NSC -derived astrocyteneuron co-culture models can be maintained at a defined (and thus reproducible) ratio of the two populations, 


\section{Neural induction $\Rightarrow$ Neural differentiation $\Rightarrow$ Co-Culture}

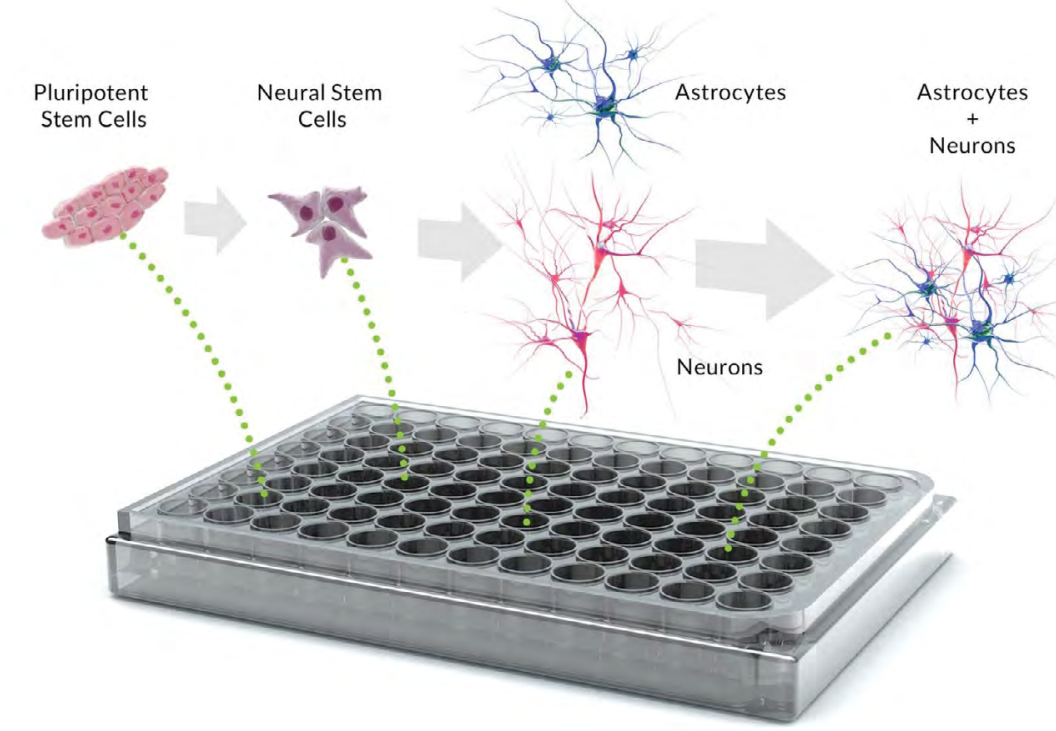

Figure 1. Neural development from pluripotent stem cells for developmental neurotoxicity screening. Neural induction of human pluripotent stem cells generates neural stem cells which can differentiate into neurons and astrocytes. Pluripotent stem cells, neural stem cells and derived neurons and astrocytes are suitable for high content developmental neurotoxicity screening.

mimicking in vivo cellular composition and interaction ${ }^{37}$. Defined ratios of human astrocytes-neuron co-culture DNT assays may be more physiologically relevant to human CNS cell composition and function than mixed cultures or solely neuronal cell cultures ${ }^{37}$. Thus, a DNT co-culture of human origin would be of great importance to the field, advancing efforts towards obtaining rapid and reliable DNT data from an in vitro CNS model (Figure 1).

\section{Conclusions}

Animal models are the current gold standard to test chemicals of unknown potency for harmful effects during developmental neurotoxicity windows of susceptibility. However, in vivo animal models are unsuitable for screening large numbers of chemicals due to high cost, low throughput, and disparities between rodent and human development. Therefore, an urgent need exists for the development of alternative test systems that will allow for faster and more cost-efficient testing of chemicals for DNT. Rodent primary cells isolated from brain tissue represent the appropriate in vivo milieu for DNT screening, yet these methods are plagued by inconsistency and a lack of species specificity. Although immortalized cell lines are of human origin, they also have their own inconsistencies and drawbacks. Therefore, hPSC-derived cell lines provide a major DNT testing advantage given their capacity for self-renewal and ability to generate the major cell types of the nervous system similar to those in vivo. Neurite outgrowth is a fundamental process of CNS development and can serve in vitro as a highly sensitive endpoint amenable to high content and throughput screening, with the potential to identify and evaluate developmental neurotoxicity and predict adverse outcomes. Because of their potentially unlimited capacity for proliferation and directed differentiation towards defined cell types, NSC are an emerging model that will bolster existing rodent studies and streamline testing on novel agents while maintaining species specificity with high reproducibility. However, the two-dimensional (2D) model platform still meets some limitation compared to three-dimensional (3D) cell culture systems since almost all cells in the in vivo environment are surrounded by other cells and extracellular matrix (ECM) in a 3D fashion. 2D cell culture does not adequately account for the natural 3D environment of cells. 3D cell culture system has gained increasing interest in drug discovery and tissue engineering due to its evident advantages in providing more physiologically relevant information and more predictive data for in vivo tests ${ }^{40}$.

In one such study, human PSC-derived endothelial cells, mesenchymal stem cells, and microglia/macrophage precursors, NSC self-assembled in vitro into 3D neural constructs with diverse populations. These 3D constructs also underwent dramatic changes in cell number, overall size, and morphology during differentiation and maturation $^{41}$. In the future, a reproducible 3D model would be suitable for predicting toxicities for chemicals that target a wide range of interactions important to brain development. 


\section{Authors' contributions}

Xian $\mathrm{Wu}$ wrote the manuscript and created table and figure. Raymond Swetenburg, Forrest Goodfellow and Steven L. Stice edited the manuscript for structure, scope and content.

\section{Acknowledgements}

The authors wish to thank Brad E. Gilleland for figure illustration editing together with Christina Elling for comments on the manuscript.

\section{Funding}

The authors wish to thank US Environmental Protection Agency EPA- R835551-STAR-F1, National Science Foundation CBET 0939511 Emergent Behavior of Integrated Cellular Systems and UGA Interdisciplinary Toxicology Program for funding support.

\section{Declaration of conflicting interests}

The author(s) declared no potential conflicts of interest with respect to the research, authorship, and/or publication of this article.

\section{References}

1. Rice D, Barone S Jr. "Critical periods of vulnerability for the developing nervous system: evidence from humans and animal models." Environ Health Perspect. 2000; 108 Suppl 3: 511-533.

2. Grandjean P, Landrigan PJ. "Developmental neurotoxicity of industrial chemicals.” Lancet. 2006; 368(9553): 2167-2178.

3. Karfunkel P. “The mechanisms of neural tube formation." Int Rev Cytol. 1974; 38(0): 245-271.

4. Northrup H, Volcik KA. "Spina bifida and other neural tube defects." Curr Probl Pediatr. 2000; 30(10): 313-332.

5. Casey BJ, Getz S, Galvan A. “The adolescent brain.” Dev Rev. 2008; 28(1): 62-77.

6. Just MA, Keller TA, Malave VL, et al. "Autism as a neural systems disorder: A theory of frontal-posterior underconnectivity." Neuroscience and Biobehavioral Reviews. 2012; 36(4): 1292-1313.

7. Bakulski KM, Rozek LS, Dolinoy DC, et al. "Alzheimer's disease and environmental exposure to lead: the epidemiologic evidence and potential role of epigenetics." Curr Alzheimer Res. 2012; 9(5): 563 573 .

8. Pei Y, Peng J, Behl M, et al. "Comparative neurotoxicity screening in human iPSC-derived neural stem cells, neurons and astrocytes." Brain Res. 2016; 1638(Pt A): 57-73.

9. Ohara R, Imamura K, Morii F, et al. "Modeling Drug-Induced Neuropathy Using Human iPSCs for Predictive Toxicology." Clin Pharmacol Ther. 2017; 101(6): 754-762.

10. Dhara S, Hasneen KK, Machacek DW, et al. "Human neural progenitor cells derived from embryonic stem cells in feeder-free cultures." Differentiation. 2008; 76(5): 454-464.

11. Dhara SK, Stice SL. "Neural differentiation of human embryonic stem cells.” J Cell Biochem. 2008; 105(3): 633-640.

12. Shin SJ, Mitalipova M, Noggle S, et al. "Long-term proliferation of human embryonic stem cell-derived neuroepithelial cells using defined adherent culture conditions." Stem Cells. 2006; 24(1): 125138.
13. Shin S, Dalton S, Stice SL. "Human motor neuron differentiation from human embryonic stem cells." Stem Cells and Development. 2005; 14(3): 266-269.

14. Majumder A, Banerjee S, Harrill JA, et al. "Neurotrophic Effects of Leukemia Inhibitory Factor on Neural Cells Derived from Human Embryonic Stem Cells." Stem Cells. 2012; 30(11): 2387-2399.

15. Saxena NK, Sharma D, Ding XK, et al. "Concomitant activation of the JAK/STAT, PI3K/AKT, and ERK signaling is involved in leptin-mediated promotion of invasion and migration of hepatocellular carcinoma cells." Cancer Research. 2007; 67(6): 2497-2507.

16. Otani T, Marchetto MC, Gage FH, et al. "2D and 3D Stem Cell Models of Primate Cortical Development Identify Species-Specific Differences in Progenitor Behavior Contributing to Brain Size." Cell Stem Cell. 2016; 18(4): 467-480.

17. Druwe I, Freudenrich TM, Wallace K, et al. "Sensitivity of neuroprogenitor cells to chemical-induced apoptosis using a multiplexed assay suitable for high-throughput screening." Toxicology. 2015; 333: 14-24.

18. Tsaneva-Atanasova K, Burgo A, Galli T, et al. "Quantifying neurite growth mediated by interactions among secretory vesicles, microtubules, and actin networks." Biophys J. 2009; 96(3): 840-857.

19. Radio NM, Mundy WR. "Developmental neurotoxicity testing in vitro: Models for assessing chemical effects on neurite outgrowth." Neurotoxicology. 2008; 29(3): 361-376.

20. Anglister L, Farber IC, Shahar A, et al. "Localization of voltagesensitive calcium channels along developing neurites: their possible role in regulating neurite elongation." Dev Biol. 1982; 94(2): 351-365.

21. Maness PF, Matten WT. "Tyrosine phosphorylation of membraneassociated tubulin in nerve growth cones enriched in pp60c-src." Ciba Found Symp. 1990; 150: 57-69; discussion 69-78.

22. Letourneau PC. "Cell-substratum adhesion of neurite growth cones, and its role in neurite elongation." Exp Cell Res. 1979; 124(1): 127138.

23. Bray D, Chapman K. "Analysis of microspike movements on the neuronal growth cone." J Neurosci. 1985; 5(12): 3204-3213.

24. Maeder CI, Shen K, Hoogenraad CC. "Axon and dendritic trafficking." Curr Opin Neurobiol. 2014; 27: 165-170.

25. Harrill JA, Freudenrich TM, Machacek DW, et al. "Quantitative assessment of neurite outgrowth in human embryonic stem cellderived hN2 (TM) cells using automated high-content image analysis." Neurotoxicology. 2010; 31(3): 277-290.

26. Harrill JA, Freudenrich TM, Robinette BL, et al. "Comparative sensitivity of human and rat neural cultures to chemical-induced inhibition of neurite outgrowth." Toxicol Appl Pharmacol. 2011; 256(3): 268-280.

27. Jeerage KM, Oreskovic TL, Hume SL. "Neurite outgrowth and differentiation of rat cortex progenitor cells are sensitive to lithium chloride at non-cytotoxic exposures." Neurotoxicology. 2012; 33(5): 1170-1179.

28. Wu X, Majumder A, Webb R, et al. "High content imaging quantification of multiple in vitro human neurogenesis events after neurotoxin exposure." BMC Pharmacol Toxicol. 2016; 17(1): 62.

29. Herculano-Houzel S. "The glia/neuron ratio: how it varies uniformly across brain structures and species and what that means for brain physiology and evolution." Glia. 2014; 62(9): 1377-1391.

30. Allen NJ, Barres BA. "NEUROSCIENCE Glia - more than just brain glue." Nature. 2009; 457(7230): 675-677.

31. Eroglu C, Barres BA. "Regulation of synaptic connectivity by glia." Nature. 2010; 468(7321): 223-231.

32. Hansebout CR, Su CX, Reddy K, et al. "Enteric glia mediate neuronal 
outgrowth through release of neurotrophic factors." Neural Regeneration Research. 2012; 7(28): 2165-2175.

33. Meyer RP, Knoth R, Schiltz E, et al. "Possible function of astrocyte cytochrome $\mathrm{P} 450$ in control of xenobiotic phenytoin in the brain: in vitro studies on murine astrocyte primary cultures." Exp Neurol. 2001; 167(2): 376-384.

34. Malaplate-Armand C, Leininger-Muller B, Batt AM. "[Astrocytic cytochromes 4 450: an enzyme subfamily critical for brain metabolism and neuroprotection]." Rev Neurol (Paris). 2004; 160(6-7): 651-658.

35. Majumder A, Dhara SK, Swetenburg R, et al. "Inhibition of DNA methyltransferases and histone deacetylases induces astrocytic differentiation of neural progenitors." Stem Cell Res. 2013; 11(1): 574-586.

36. Shaltouki A, Peng J, Liu QY, et al. "Efficient Generation of Astrocytes from Human Pluripotent Stem Cells in Defined Conditions." Stem Cells. 2013; 31(5): 941-952.

37. Wu X, Yang X, Majumder A, et al. "From the Cover: AstrocytesAre
Protective Against Chlorpyrifos Developmental Neurotoxicity in Human Pluripotent Stem Cell-Derived Astrocyte-Neuron Cocultures." Toxicol Sci. 2017; 157(2): 410-420.

38. Jones EV, Cook D, Murai KK. "A neuron-astrocyte co-culture system to investigate astrocyte-secreted factors in mouse neuronal development." Methods Mol Biol. 2012; 814: 341-352.

39. Fukushima K, Miura Y, Sawada K, et al. "Establishment of a Human Neuronal Network Assessment System by Using a Human Neuron/ Astrocyte Co-Culture Derived from Fetal Neural Stem/Progenitor Cells." J Biomol Screen. 2016; 21(1): 54-64.

40. Edmondson R, Broglie JJ, Adcock AF, et al. "Three-Dimensional Cell Culture Systems and Their Applications in Drug Discovery and CellBased Biosensors." Assay and Drug Development Technologies. 2014; 12(4): 207-218.

41. Schwartz MP, Hou Z, Propson NE, et al. "Human pluripotent stem cellderived neural constructs for predicting neural toxicity." Proc Natl Acad Sci U S A. 2015; 112(40): 12516-12521. 